\title{
Balancing of an Inverted Pendulum with a SCARA Robot
}

\author{
Bernhard Sprenger ${ }^{1)}$, Ladislav Kucera ${ }^{1)}$, and Safer Mourad ${ }^{2)}$ \\ 1) Swiss Federal Institute of Technology Zurich (ETHZ) • Institute of Robotics \\ 8092 Zurich, Switzerland • email: sprenger@ifr.mavt.ethz.ch, kucera@ifr.mavt.ethz.ch \\ 2) Seestrasse 64, 9326 Horn, Switzerland • email: hmos@lah.leica.ch
}

\begin{abstract}
The balancing of an inverted pendulum with a robot is a good example for analyzing fast sensor guided movements and testing modern control strategies. This classical benchmark is difficult to achieve as it attempts to stabilize a system that is unstable in its open loop configuration. Additionally, there are sensing problems (measuring very small angles) and nonlinear effects in the actuators (friction and elasticity of the gears).

The pendulum is projected to the $x z$ - and yz-plane of the inertial coordinate system. These projections are treated independently from each other and are controlled individually by a state space controller in the $x$-and y-axis respectively. The nonlinearities of the robot are compensated by using inverse dynamics and inverse kinematics.

A specially developed sensor system allows the contactless measurement of the angles. This system consists of a small magnet, placed at the bottom of the pendulum, and hall effect sensors placed inside the end effector.
\end{abstract}

\section{Introduction}

A robot balancing an inverted pendulum is an impressive demonstration object, that shows the performance achieved by an intelligent combination of modern control algorithms, robotics and electronics. Therefore it is a typical product emerging from mechatronics aspects. The system realization requires the design of a state space controller, calculation of the inverse kinematics and dynamics, and the development of specialized signal processing electronics due to measure the inclination angle of the pendulum.

The following single pendulum setups with increasing requirements to the control task are known:

- A pendulum with 1 dof (degree of freedom) mounted on a base rotating around the $z$-axis [1]. This setup is similar to the classical one described below but the direct drive allows higher accelerations with less friction effects.

- A pendulum with 1 dof mounted on a linear slide [2],[3],[4]. This is the classical setup with a well known mechanical model.
- A pendulum with 2 dof mounted on a robot with decoupled or weak coupled links [5]. At least one link can be moved without affecting the position of the other link. The separation of the balancing planes can be done easily.

- A pendulum with 2 dof mounted on a robot with strongly coupled links. The balancing planes have to be separated in order to get a reasonable controller order. But in contrast to previous setup the remaining nonlinear coupling terms require a more robust control system.

In publications, the balancing of the inverted pendulum is often used as plant for modern control strategies such as fuzzy control [1], high-and-low gain approach [2], nonlinear control [4] and neural networks [5]. These modern strategies often base on linear control structures but enclose overlaid auto-tuning or adaptive controllers.

Either control of the stable or the instable equilibrium position can be done. It is obvious, that the control of an openloop instable system is more difficult than the control of a stable one. However control of a stable pendulum is useful in view of good transient behavior. Such a control takes part of industrial applications, for example the control of cranes [6]. Additional difficulties are given with a second degree of freedom and when rotational drives instead of linear drives are used.

The system described in this paper is based on a SCARA robot Fig. 1. Due to this setup the balancing planes are strongly coupled. Additionally the robot uses harmonic drive joints and therefore its drives have high friction and elasticity. All this nonlinearities of the system are difficult to model. The goal of this paper is to compensate as many as possible of these nonlinearities and to design a robust linear control.

Furthermore a unique sensor system has been developed, allowing a contactless and invisible measurement of the angles of the pendulum. The sensor system is built up with hall effect sensors. 


\section{Experimental Setup}

The robot is a SCARA type one with 2 dof. It is controlled by a VME-Bus system based on a 68040 processor and uses two 90W DC motors coupled with harmonic drive ${ }^{1}$ gears as actuators. The complete controller software is written in Oberon $^{2}$, using XOberon as cross development system.

The pendulum can be placed on the end effector without any attachment. Its angles are measured by hall effect sensors placed inside of the end effector.

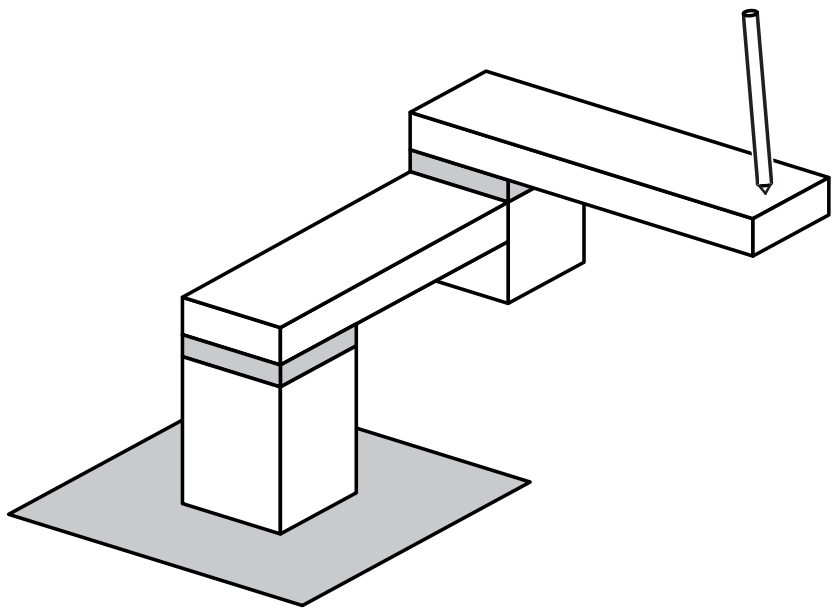

Fig. 1: Experimental setup - SCARA robot balancing the inverted pendulum.

\section{Mechanical Model}

\subsection{Pendulum}

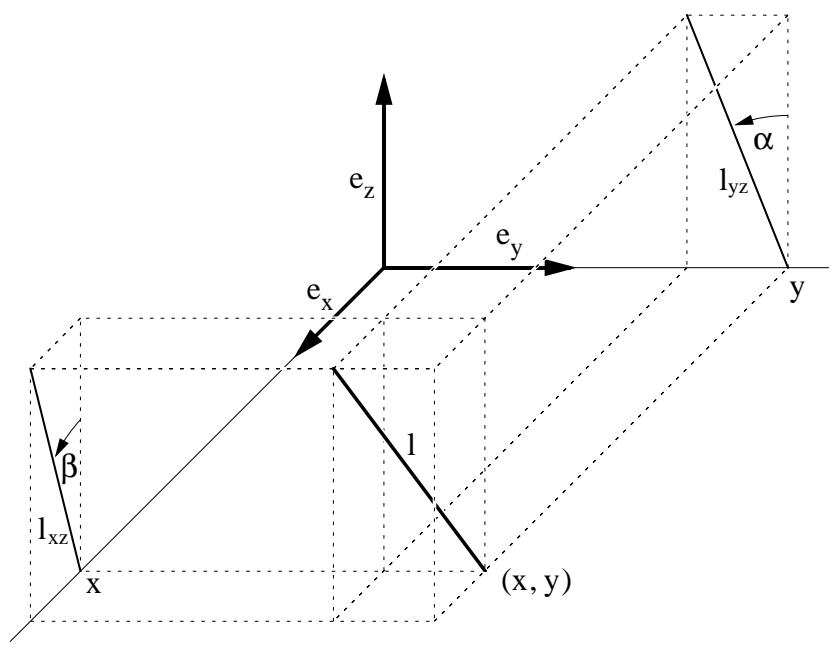

Fig. 2: Projection of the pendulum to the $x z$ - and yz-plane.

The pendulum is projected to the $x z$ - and $y z$-plane of the inertial coordinate system as shown in Fig. 2. These projections are considered as coupled pendulums inside two orthogonal planes. Their inertias $J_{x z}, J_{y z}$ can be described

1. Harmonic drives are light, free from play and have a high ratio (50-200) but with high friction and elasticity

2. Oberon is an object oriented programing language developed at the ETH as functions of their lengths $l_{x z}, l_{y z}$ or their inclination angles $\alpha, \beta$ with respect to the origin.

$$
\begin{aligned}
& J_{x z}=J \cdot \frac{l_{x z}{ }^{2}}{l^{2}}=J \cdot \frac{1}{\frac{\cos (\beta)^{2}}{\cos (\alpha)^{2}}+\sin (\beta)^{2}} \\
& J_{y z}=J \cdot \frac{l_{y z}^{2}}{l^{2}}=J \cdot \frac{1}{\frac{\cos (\alpha)^{2}}{\cos (\beta)^{2}}+\sin (\alpha)^{2}}
\end{aligned}
$$

Assuming that the range of $\alpha$ and $\beta$ is within $\pm 10^{\circ}$, the variation of these inertias is less than $6.3 \%$. Therefore the variations of the following parameters are substitutable:

$$
\begin{aligned}
& l_{x z} \cong l_{y z} \cong l \\
& J_{x z} \cong J_{y z} \cong J
\end{aligned}
$$

After this simplification the projections are considered as two uncoupled pendulums in different planes, which can be controlled individually. The calculation of the motion eqns. (5), (6) for planar pendulums is straight forward and has been carried out in the literature, so it is not necessary to repeat this task.

$$
\begin{aligned}
& \ddot{\alpha}=\frac{m \cdot g}{2 \cdot J} \cdot(g \cdot \sin (\alpha)+\ddot{y} \cdot \cos (\alpha)) \\
& \ddot{\beta}=\frac{m \cdot g}{2 \cdot J} \cdot(g \cdot \sin (\beta)+\ddot{x} \cdot \cos (\beta))
\end{aligned}
$$

Assuming that $\alpha$ and $\beta$ are small, these two eqns. (5), (6) can be linearized. Finally the following reduced equations (7), (8) can be obtained.

$$
\begin{aligned}
& \ddot{\alpha}=\frac{m \cdot g}{2 \cdot J} \cdot(g \cdot \alpha+\ddot{y}) \\
& \ddot{\beta}=\frac{m \cdot g}{2 \cdot J} \cdot(g \cdot \beta+\ddot{x})
\end{aligned}
$$

Symbols:

$\begin{array}{ll}-\mathrm{m} & : \text { Mass of the pendulum } \\ -1 & : \text { Length of the pendulum } \\ -\mathrm{J} & : \text { Inertia of the pendulum } \\ -\mathrm{g} & : \text { Acceleration due to gravity }\left(9.81 \mathrm{~ms}^{-2}\right)\end{array}$

\subsection{Robot}

The model of the robot consists of the geometrical properties of the machine (lengths of the arms: $l_{1}, l_{2}$; centers of gravity of the arms: $l_{s 1}, l_{s 2}$ ), the inertias $J_{1}, J_{2}$ and the masses $m_{1}, m_{2}$ of the arms as shown in Fig. 3. With respect to the high transmission ratio $n_{1}, n_{2}$ of the gears, the inertias $J_{r 1}, J_{r 2}$ of the drives can't be neglected and therefore are part of the model too. A strong friction requires an accurate model of the gears and is modeled with two nonlinear functions $b_{1}=b_{1}\left(\dot{q}_{1}, \ddot{q}_{1}\right) \quad$ and 
$b_{2}=b_{2}\left(\dot{q}_{2}, \ddot{q}_{2}\right)$. The friction parameters are determined in the following chapter.

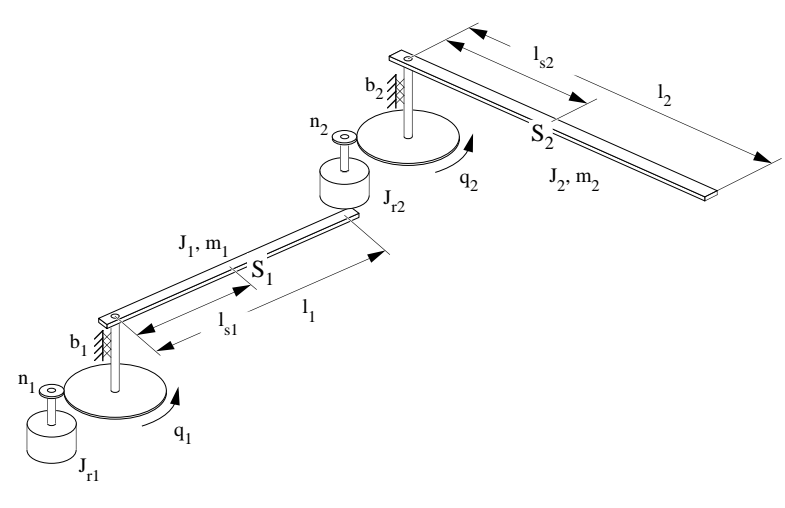

Fig. 3: Model of the robot.

The kinematics of this robot are expressed by the following two equations:

$$
\begin{aligned}
& x=l_{1} \cos \left(q_{1}\right)+l_{2} \cos \left(q_{1}+q_{2}\right) \\
& y=l_{1} \sin \left(q_{1}\right)+l_{2} \sin \left(q_{1}+q_{2}\right)
\end{aligned}
$$

The inverse kinematics for the joint accelerations $\ddot{q}$ are evaluated from the equations (9) and (10). The result is a function of the joint angles $\underline{q}$, the joint velocities $\dot{q}$ and the accelerations in $x$ - and $y$-direction.

$$
\ddot{q}=\operatorname{Inv} \operatorname{KinAcc}(\underline{q}, \dot{q}, \ddot{x}, \ddot{y})
$$

The dynamics of the robot is calculated by using the method of Lagrange. It can be represented by the following notation:

$$
\underline{M}(\underline{q}) \cdot \underline{\ddot{q}}+\underline{V}(\underline{q}, \underline{\dot{q}})=\tau_{-}-\underline{F}(\underline{q}, \underline{\dot{q}})
$$

where:

- $M(q) \quad$ : Mass matrix

- $\underline{V}(\underline{q}, \underline{q}) \quad$ : Coriolis and centrifugal terms

- $F(\underline{q}, \underline{\dot{q}}) \quad$ : Friction, elasticity and damping terms

- $\bar{\tau} \quad$ : Vector of joint forces, torques

\subsection{Friction}

The use of harmonic drives allows to build small, light and high ratio gears but they deliver an unpleasant friction. This frictional force is heavily dependent on the actual gear's position and the history of the gear's movement. In order to model the friction, it was measured by applying a constant torque to the joints. After a few seconds a constant velocity has ensued. The relation between torque and velocity of the gear was recorded several times and is shown in Fig. 4.

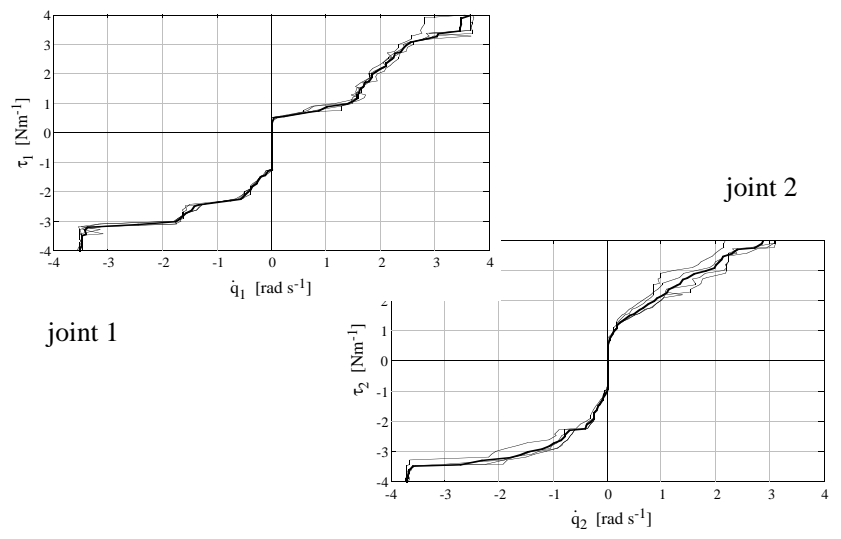

Fig. 4: Friction measurements.

These measurements show some of the bad consistency, therefore the mean value is taken from these measurements for each joint. Afterwards the resulting curve is approximated by a polygon composed of 5 straight lines as shown in Fig. 5.

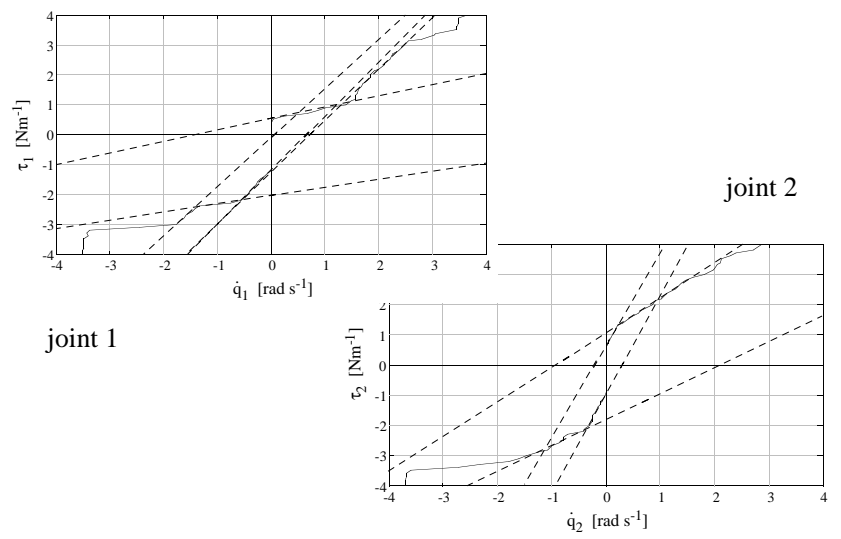

Fig. 5: Approximation of the friction.

These approximations are used in the dynamic compensation. The result of this model based compensation is shown in Fig. 7.

\section{Control Strategy}

A linear controller design requires a sufficiently linearized system. The use of a SCARA robot, which has strongly coupled joints, needs an adequate compensation inside the full work space. The geometrical nonlinearities are compensated with the inverse kinematics; the remaining nonlinearities, due to the coupling of the joints, are reduced by the use of the following dynamic compensation.

\subsection{Dynamic Compensation of the Robot}

The dynamics of the robot can be approximated with the following motion equation:

$$
\underline{\tau}=\underline{\tilde{M}}(\underline{q}) \cdot \underline{\ddot{q}}_{s}+\underline{\tilde{V}}(\underline{q}, \underline{\dot{q}})+\underline{\tilde{F}}(\underline{q}, \underline{\dot{q}})
$$


Assuming that Equation (13) represents an adequate model of the robot, the dynamic nonlinearities can be compensated as shown in the block diagram of Fig. 6.

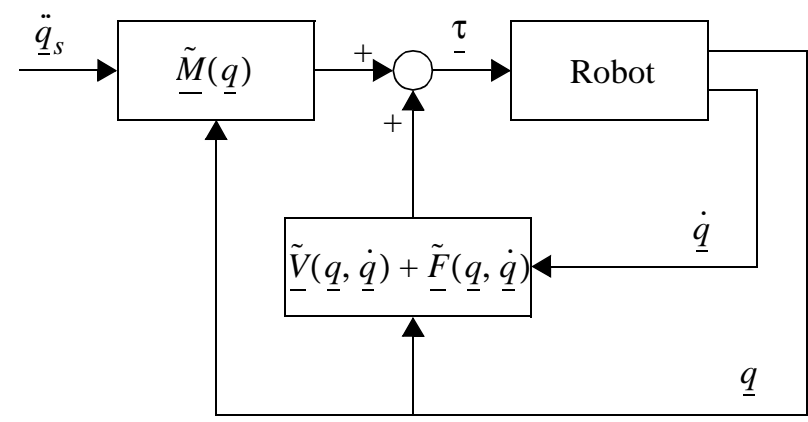

Fig. 6: Dynamic compensation.

Applying this block diagram leads to a system with $\underline{\ddot{q}}_{s}$ as system input and the complete nonlinear system can be approximated as shown in (14).

$$
\underline{q} \cong \underline{q}_{S}
$$

Fig. 7 shows a measurement of the joint accelerations $\ddot{q}$ and the inputs $\underline{\ddot{q}}_{s}$ of the dynamic compensation. It can be seen, that the mean deviation between $\underline{q}$ and $\underline{q}_{s}$ is smaller than $10 \%$.
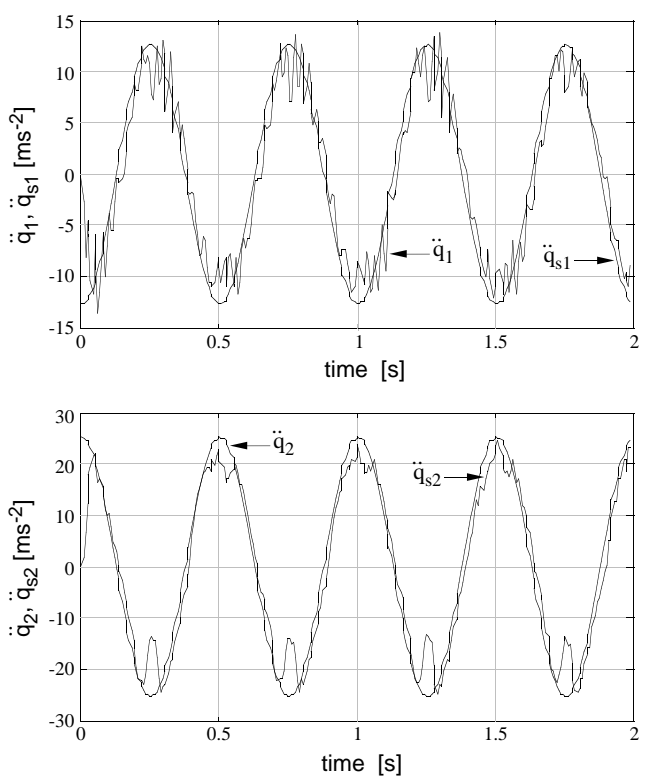

Fig. 7: Result of the dynamic compensation.

There are mainly two reasons for the remaining differences of the curves. First, there are unmodeled coupling effects from one joint to the other one. Second, friction effects at zero velocity of the joints are very noisy, which results in a high frequency oscillation of the measured joint accelerations. As it is difficult to improve the model of the friction, the curves of Fig. 7 must be seen as the limit, what can be reached with the dynamic compensation. Further work will be done, where a control loop tries to minimize the difference between desired and real acceleration. Therefore the joint accelerations are measured with acceleration sensors. Preliminary results of such a setup have shown, that the precision can be improved by a factor of two.

\subsection{Control Structure}

As seen in chap. 3.1. the two-dimensional pendulum can be approximated by two one-dimensional pendulums projected into two cartesian planes ( $x z$-plane and $y z$-plane). Each one-dimensional pendulum has its own controller. The outputs of the controllers are the desired accelerations $\ddot{x}_{s}$ and $\ddot{y}_{s}$ for the origin of the pendulum. The inverse kinematics (11) converts these accelerations into desired joint accelerations $\ddot{q_{s 1}}$ and $\ddot{q_{s 2}}$, which are used by the dynamic compensation (Fig. 6) to calculate the desired joint torques $\tau_{1}$ and $\tau_{2}$ of the robot. The complete system structure is shown in Fig. 8.

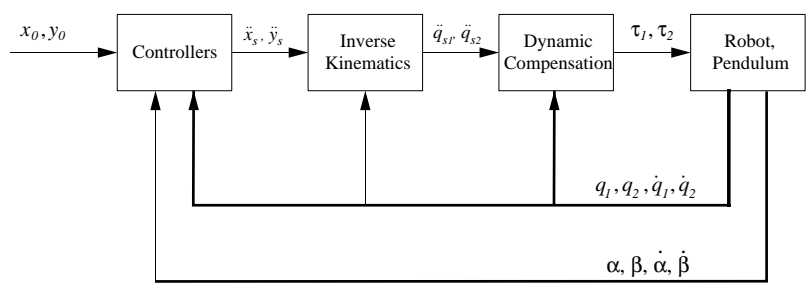

Fig. 8: Closed loop control system.

\subsection{State space controller}

As the robot is linearized with the inverse kinematics and with the dynamic compensation, the motion eqns. (7) and (8) represent an adequate control system description of the plant. In Fig. 9 the structure of the plant and of a state space controller is shown. With respect to the identical structure of both controllers, in the following only the controller for the pendulum inside the $x z$-plane will be discussed.

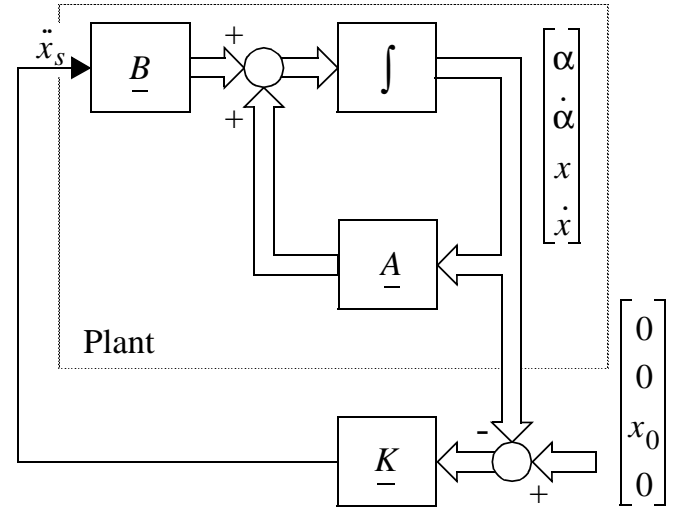

Fig. 9: State space controller of an one-dimensional pendulum.

The control of the states $\alpha$ and $\dot{\alpha}$ would be sufficient for the balancing of a pendulum. Due to the restricted work 
space, the states $x$ and $x$ have to be controlled too. This leads to a state space representation of 4 th order as shown in (15).

$$
\begin{aligned}
& \underline{\dot{X}}=\underline{A} \cdot \underline{X}+\underline{B} \cdot \ddot{x}_{s} \\
& =\underline{K} \cdot\left(\underline{X_{0}}-\underline{X}\right)
\end{aligned}
$$

where:

$$
\underset{A}{A}=\left[\begin{array}{cccc}
0 & 1 & 0 & 0 \\
\frac{m l g}{2 J} & 0 & 0 & 0 \\
0 & 0 & 0 & 1 \\
0 & 0 & 0 & 0
\end{array}\right] \underline{B}=\left[\begin{array}{c}
0 \\
\frac{m l}{2 J} \\
0 \\
1
\end{array}\right] \quad \underline{X}=\left[\begin{array}{c}
\alpha \\
\dot{\alpha} \\
x \\
\dot{x}
\end{array}\right] \quad \underline{X}_{0}=\left[\begin{array}{c}
0 \\
0 \\
x_{0} \\
0
\end{array}\right]
$$

The 4th order controller is realized as a state space controller with the feedback matrix $K$. The design method was a Ricatti (LQR). The controller needs to be designed in such a way, that the time response of the inclination angle $\alpha$ of the pendulum has higher dynamics than the time response of the robot position $x$. But it should be noticed, that a very slow dynamics of the robot position let the robot reach its work space limits. The optimal dynamics of these two parts has to be found experimentally.

The stationary behavior of the inclination angle $\alpha$ visualized in the phase plane $(\alpha, \dot{\alpha})$ is a stable limit cycle. Stability is given, when the trajectory is rotating into the right side. The diameter of the limit cycle is related to the quantization of the measurement of the angle $\alpha$, i.e. higher resolution can improve the control quality.

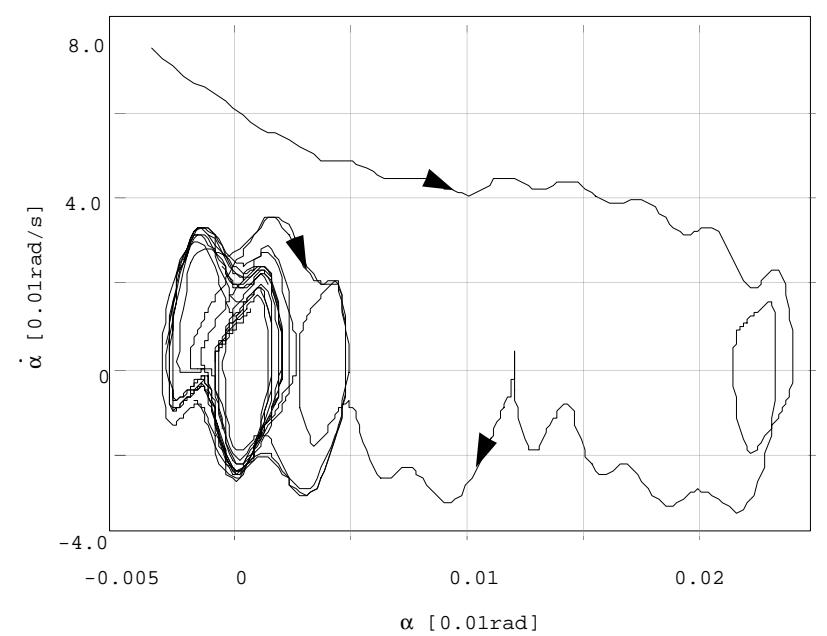

Fig. 10: Phaseplane of $\alpha$, stable limit cycle with an angleresolution of $0.003 \mathrm{rad}$.

\section{Sensors}

One of the elementary problems to solve was the measurement of the two angles of the pendulum. A sensor is required, which measures absolute angles, which gives a good resolution for very small angular displacements and which is not fixed to the pendulum.
Absolute measurement of the angle is desirable, because there is normally no reference to calibrate the sensor. A relative measuring system such as an incremental encoder needs a start up procedure to define the zero position of the angle.

Within a range of the sensor must have an acceptable resolution. The controller provides the derivative of this angular signal for estimating the rotational velocity of the pendulum. With a low resolution of the angular measurement, the resolution of the velocity becomes very bad and the controller will not be able to stabilize the pendulum.

Measurement systems such as incremental encoders or potentiometers would be fixed to the pendulum. Technically this would not cause any problems. For an observer however, it might appear unclear, how the system works. Often the question was asked, if this thing - the incremental encoder - which looks like a motor, is holding the pendulum vertically! The show is much better, when the pendulum is not fixed to the robot.

During the development of the system, three types of sensors were tested and the following observations were made:

\subsection{Potentiometer}

It was easy to build a two dimensional measurement system, as a normal joystick could be modified. The electrical signal output from the potentiometer for small angles was very noisy and the controller was not able to balance the pendulum. Therefore this measuring method was not used.

\subsection{Encoder}

The best controller performance (robustness and diameter of the limit cycle) was reached with a high resolution encoder (4096 pulses per ). The large disadvantage of this method is the painful start-up procedure while finding the equilibrium position of the pendulum.

The resolution of the angular velocity is low, but can be increased with special hardware, which allows the interpretation of the period of time from one encoder pulse to the next one.

\subsection{Hall Sensor}

A permanent magnet, placed inside the pendulum, generates a static magnetic flux field. In the case of static fields, all aluminum parts behave nonmagnetically as does the air and don't damp the flux distribution. The flux lines which cut the sensor produce an electrical signal. A differential evaluation of the two hall effect sensors gives a signal proportional to the inclination angle.

The signal quality of the hall effect sensor is not as good as from an incremental encoder, but good enough to balance the pendulum. The free standing nature of the measuring 
system gives the impression, that the pendulum is really balanced by the robot.

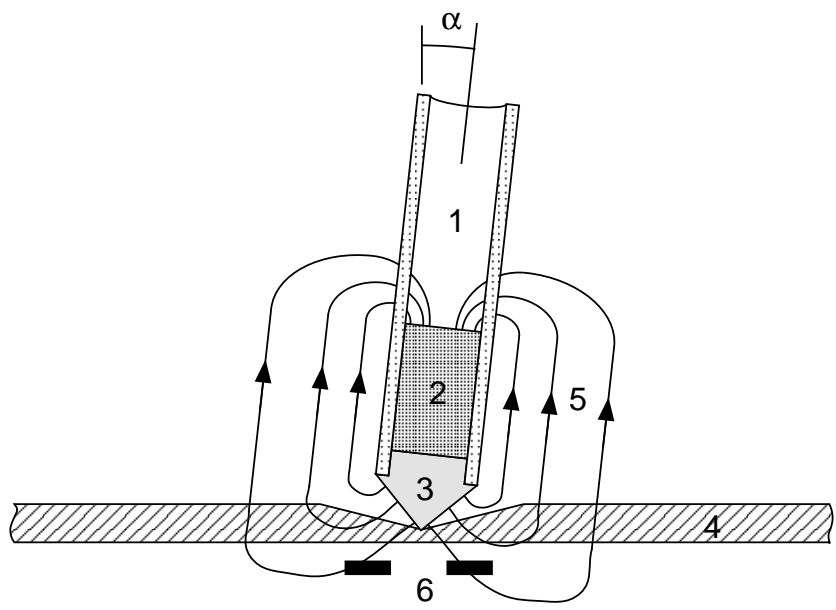

Fig. 11: Cross section of pendulum and sensor.

$$
\begin{array}{ll}
\text { - 1: } & \text { pendulum (aluminum tube) } \\
\text { - 2: } & \text { permanent magnet } \\
\text { - 3: } & \text { vertex (steel) } \\
\text { - 4: } & \text { mounting plate (aluminum) } \\
\text { - 5: } & \text { magnetic flux } \\
\text { - } 6: & \text { two hall effect sensors } \\
\text { - } \alpha: & \text { inclination angle of the pendulum }
\end{array}
$$

The contactless mounting of the pendulum has one disadvantage. When the robot or the floor vibrates, the pendulum tends to jump out of its notch.

\section{Conclusions}

The goal of the work described in this paper is the theoretical analysis and the practical realization of a robot balancing a 2 dof pendulum. The setup robot with two rotational axes and a 2 dof pendulum is unique. Comparable experiments are limited to linear drives or the balance of a 1 dof pendulum.

Based on a useful system reduction, a compensator for the robot's and the pendulum's nonlinearities could be implemented, i.e. the forward and inverse kinematics, the compensation of the dynamics and the gear frictions are calculated at each sampling interval by the controller. Finally a robust working experimental apparatus was realized.

The sensing problem of measuring the inclination angle of the pendulum was solved by using a contactless measuring setup based on hall effect sensors inside the end effector and a permanent magnet inside the pendulum.

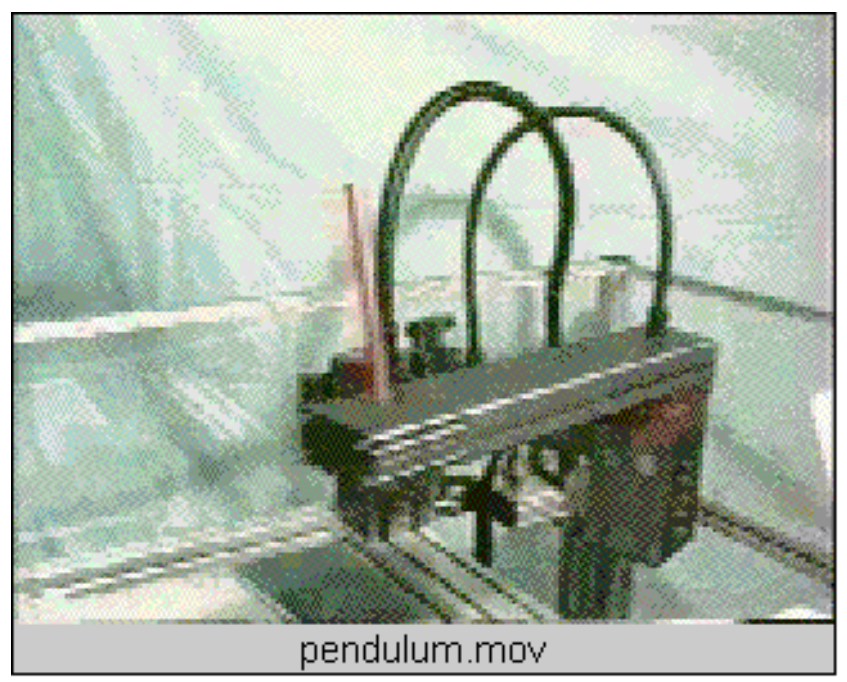

Fig. 12: Movie of the Inverted Pendulum

\section{References}

[1] S. Yurkovich and M. Widjaja, Fuzzy Controller Synthesis for an Inverted Pendulum System, Control Eng. Practice, Vol. 4, No. 4, pp. 445-469, 1996.

[2] Z. Lin, A. Saberi, M. Gutmann and Y. Shamash, Linear Controller for an Inverted Pendulum Having Restricted Travel - A High-and-Low Gain Approach, Proceedings of the American Control Conference, Seattle, pp. 2980-2984, June 1995.

[3] Q. Wei, W. P. Dayawansa and W. S. Levine, Nonlinear Controller for an Inverted Pendulum Having Restricted Travel, Automatica, Vol. 31, No. 6, pp. 841-850, 1995.

[4] C. C. Chung and J. Hauser, Nonlinear Control of a Swinging Pendulum, Automatica, vol. 31, No. 6, pp. 851-862, 1995.

[5] J. Nelson and L. G. Kraft, Real-Time Control of an Inverted Pendulum System using Complementary Neural Network and Optimal Techniques, Proceedings of the American Control Conference, Baltimore, pp. 2553-2554, June 1994.

[6] K. Gierlotka, B. Grzesik, A. Nowak and J. Wojnarowski, Control of overhead crane drive with centred motion and elimination of the Bevel, IEEEInternational-Symposium-on-Industrial-Electronics, vol 2, pp. 1061-1065, 1996.

[7] W. Maletinsky, M.F. Senning and F. Wiederkehr, Observer Based Control of a Double Pendulum, Proceedings of IFAC World Congress, Kyoto, 1981. 